\title{
A relativistic Glauber approach to polarization transfer in ${ }^{4} \mathrm{He}\left(\vec{e}, e^{\prime} \vec{p}\right)$
}

\author{
P. Lava, ${ }^{1}$ J. Ryckebusch, ${ }^{1}$ and B. Van Overmeire, ${ }^{1}$ \\ ${ }^{1}$ Department of Subatomic and Radiation Physics, Ghent University, Belgium
}

December 23, 2018

\begin{abstract}
Polarization-transfer components for ${ }^{4} \mathrm{He}\left(\vec{e}, e^{\prime} \vec{p}\right)^{3} \mathrm{H}$ are computed within the relativistic multiplescattering Glauber approximation (RMSGA). The RMSGA framework adopts relativistic singleparticle wave functions and electron-nucleon couplings. The predictions with free and various parametrizations for the medium-modified electromagnetic form factors are compared to the world data.
\end{abstract}

In conventional nuclear physics nuclei are described in terms of point-like protons and neutrons, interacting through the exchange of mesons. It has been a long-standing and unresolved issue whether the electromagnetic properties of bound nucleons differ from those of free nucleons. Exclusive $A\left(\vec{e}, e^{\prime} \vec{p}\right)$ measurements have been put forward as a tool to investigate the possible modifications attributed to the presence of a medium. In polarized electron free-proton scattering, the ratio of the electric $\left(G_{E}\left(Q^{2}=-q^{\mu} q_{\mu}\right)\right)$ to the magnetic $\left(G_{M}\left(Q^{2}\right)\right)$ Sachs form factors, can be extracted from [1]

$$
\frac{P_{x}^{\prime}}{P_{z}^{\prime}}=-\frac{G_{E}\left(Q^{2}\right)}{G_{M}\left(Q^{2}\right)} \frac{2 M_{p}}{E_{e}+E_{e^{\prime}}} \tan ^{-1}\left(\frac{\theta_{e}}{2}\right) .
$$

Here, $q^{\mu}$ is the four-momentum transfer, $P_{x}^{\prime}$ and $P_{z}^{\prime}$ is the transferred polarization in the direction perpendicular to and parallel with the three-momentum transfer, and $\theta_{e}$ the electron scattering angle. For bound nucleons, deviations from the measured ratio of $P_{x}^{\prime} / P_{z}^{\prime}$ from the above value ( thereby adopting free-nucleon form factors) can indicate the existence of medium modifications. Finding signatures of medium modifications, however, requires an excellent control over all those ingredients of the $A\left(\vec{e}, e^{\prime} \vec{p}\right)$ reaction process that are directly related to the presence of a nuclear medium, such as final-state interactions (FSI), meson-exchange currents (MEC) and isobar currents (IC). Of all observables accessible in $A\left(\vec{e}, e^{\prime} \vec{p}\right)$, the transferred polarization components have been recognized as the ones with the weakest sensitivity to FSI, MEC and IC distortions. Recently, ${ }^{4} \mathrm{He}\left(\vec{e}, e^{\prime} \vec{p}\right)$ data have been reported [2, 3], covering the range $0.4 \leq Q^{2} \leq 2.6(\mathrm{GeV} / \mathrm{c})^{2}$. This kinematic regime may outreach the range of applicability of optical-potential approaches for describing FSI mechanisms. Indeed, given the highly inelastic and diffractive nature of proton-nucleon scattering at proton lab momenta exceeding $1 \mathrm{GeV} / \mathrm{c}$, the use of optical potentials seems rather unnatural and Glauber multiple-scattering theory provides a more natural and economical description of FSI mechanisms [4. Recently, we developed an unfactorized and relativistic version and dubbed it the relativistic multiple-scattering Glauber approximation (RMSGA) [5]. In Ref. [6], numerical calculations for the polarization-transfer components in ${ }^{4} \operatorname{He}\left(\vec{e}, e^{\prime} \vec{p}\right)$ are performed with both free and medium-modified electromagnetic form factors. For the latter we used the 


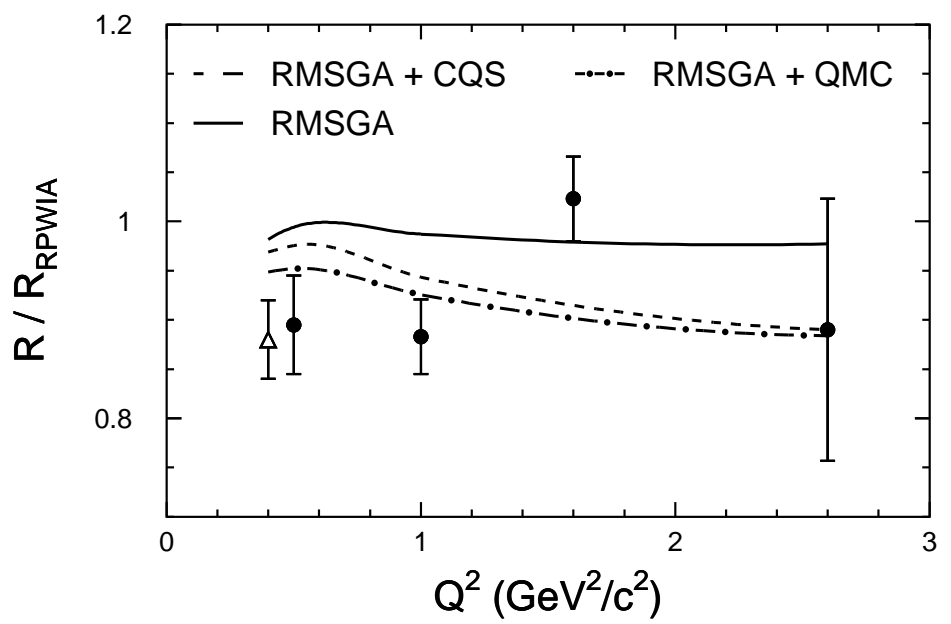

Figure 1: The superratio $R / R_{R P W I A}$ as a function of $Q^{2}$ in ${ }^{4} \mathrm{He}$. The solid curve shows RMSGA predictions using free-proton electromagnetic form factors. The dotted (dotdashed) curve represents RMSGA calculations with in-medium electromagnetic form factors from the CQS (QMC) model. Data are from Refs. 2] (open triangle) and [3] (solid circles).

predictions of the quark-meson coupling model (QMC) [7]. In this contribution we use alternative predictions of the chiral quark-soliton model (CQS) [8]. The CQS nucleon model puts more emphasis on the role of the sea than the QMC framework. As a result, the value of the magnetic moment remains practically unchanged.

In Figure 1, the ${ }^{4} \mathrm{He}$ polarization-transfer results are expressed in terms of a double ratio $R$

$$
R=\frac{\left(P_{x}^{\prime} / P_{z}^{\prime}\right)_{4} H e}{\left(P_{x}^{\prime} / P_{z}^{\prime}\right)_{1} H},
$$

with the relativistic plane-wave impulse approximation (RPWIA) result as baseline. Substituting the free forms factors with the CQS ones reduces R. At $Q^{2} \leq 1(\mathrm{GeV} / \mathrm{c})^{2}$ the reductions are smaller than those observed for the computed values of the QMC model. At higher $Q^{2}$, both models predict very similar effects. A better overall description of the data is obtained with the medium-modified form factors.

\section{References}

[1] R.G. Arnold, C.E. Carlson, and F. Gross, Phys. Rev. 23 (1981) 363

[2] S. Dieterich et al., Phys. Lett. B 500 (2001) 47

[3] S. Strauch et al., Phys. Rev. Lett. 91 (2003) 052301

[4] R. Glauber, and G.Matthiae, Nucl. Phys. B 21 (1970) 135

[5] J. Ryckebusch, D. Debruyne, P. Lava, S. Janssen, B. Van Overmeire, and T. Van Cauteren, Nucl. Phys. A 728 (2003) 226

[6] P. Lava, J. Ryckebusch, B. Van Overmeire, and S. Strauch, Phys. Rev. C 71 (2005) 034604

[7] D.H. Lu, A.W. Thomas, K. Tsushima, A.G. Williams, and K. Saito, Phys. Lett. B 417 (1998) 217

[8] J.R. Smith and G.A. Miller, Phys. Rev. C 70 (2004) 065205 